Revue

Revue de l'histoire des religions

de Ihistoire des religions

3 | 2016

Varia

\title{
Pierre-Henry DE BRUYN, Le taoïsme. Chemins de
}

découverte

Paris, CNRS Éditions (« Biblis »), 2014

Kyong-Kon Kim

\section{CpenEdition}

Journals

Édition électronique

URL : http://journals.openedition.org/rhr/8593

DOI : $10.4000 /$ rhr.8593

ISSN : 2105-2573

Éditeur

Armand Colin

Édition imprimée

Date de publication : 1 septembre 2016

Pagination : 428-431

ISBN : 978-2-200-93061-5

ISSN : 0035-1423

Référence électronique

Kyong-Kon Kim, «Pierre-Henry de BRuYn, Le taoïsme. Chemins de découverte », Revue de l'histoire des religions [En ligne], 3 | 2016, mis en ligne le 07 octobre 2016, consulté le 23 septembre 2020. URL http://journals.openedition.org/rhr/8593; DOI : https://doi.org/10.4000/rhr.8593

Ce document a été généré automatiquement le 23 septembre 2020

Tous droits réservés 


\title{
Pierre-Henry DE BRUYN, Le taoïsme. Chemins de découverte
}

Paris, CNRS Éditions (« Biblis »), 2014

\author{
Kyong-Kon Kim
}

\section{RÉFÉRENCE}

Pierre-Henry DE BRUYN, Le taoïsme. Chemins de découverte, Paris, CNRS Éditions (« Biblis »), 2014, 18 cm, 282 p., $10 €$, ISBN 978-2-271-08258-9.

Bien que le taoïsme reflète de manière authentique la religiosité chinoise autochtone, la recherche académique, à cet égard, demeure jusqu'à présent, contrairement à l'attention portée sur le confucianisme et le bouddhisme chinois et à l'exception de lectures philosophiques et herméneutiques du Daode jing et du Zhuangzi, plutôt réservée à un nombre restreint de spécialistes tant en Orient qu'en Occident. Quant au grand public occidental, s'il prend connaissance fortuitement de certains aspects cosmoanthropologiques taoïques par l'intermédiaire de pratiques thérapeutiques telles que le taiji quan, le qigong, le daoyin et le zhenjiu (acupuncture et moxibustion), l'accès aux rites religieux taoïstes, lui, s'avère encore moins aisé.

Le présent ouvrage, issu des conférences universitaires données en 2006 par P.-H. de Bruyn, sinologue à l'Université de La Rochelle, publié initialement en 2009 et intégré dans la collection «Biblis » $\left(\mathrm{n}^{\circ} 94\right)$ en tant que deuxième opus relatif au taoïsme, suite à celui d'Isabelle Robinet intitulé Histoire du taoïsme. Des origines au XIVe siècle $\left(\mathrm{n}^{0} 23,2012\right.$; éd. orig. 1991), vise, en effet, tout en contribuant à la compréhension de l'histoire du taoïsme dans sa dimension polymorphe, à susciter un intérêt plus large pour l'étude daologique, notamment à travers un exposé des visions du corps humain, considéré par l'ensemble des taoïstes comme le lieu privilégié de la réalisation ultime de l'homme.

3 Tout d'abord, l'auteur identifie dans les deux livres antiques placés au commencement du taoïsme, le Daode jing et le Zhuangzi (ch. I), des éléments fondamentaux taoïques pour la compréhension du corps. Si, dans le premier, le corps, une fois libéré de toute 
agitation psychique et sociétale, se révélait comme le lieu privilégié d'expérience de la puissance du Dao, il ne serait, d'après le deuxième, autre qu'un lieu privilégié pour cultiver la vie et retrouver l'unité avec le Dao, l'origine même de toute réalité, car l'homme parviendrait, par la pratique de la méditation assise 坐忘, du jeûne du cœur 心齊 et des régimes alimentaires spécifiques, à l'état d'homme véritable 眞人 ou immortel 仙人. Puis, le sinologue relève deux événements majeurs survenus au II I $^{\mathrm{e}}$ siècle de l'ère chrétienne, quelques siècles après cette phase initiale: dans le cadre de l'exorcisme pratiqué au sein du mouvement des maîtres célestes, Tianshi dao 天師道 (ch. II), une des premières communautés taoïstes religieuses, le corps fut considéré comme un corps familial, c'est-à-dire porteur des mémoires transgénérationnelles; le livre dit de la grande paix, Taiping jing 太平經 (ch. III), contenant une vision du monde harmonieux basée sur la théorie des cinq mouvements, wuxing shuo 五行說, atteste, à l'intérieur du corps humain, la présence de plusieurs divinités qui assurent son bon fonctionnement ainsi que de trois parasites, porteurs de la mort. Quant à l'ouvrage de Ge Hong (ch. Iv), intitulé Baopuzi 抱樸子 (vers 320), il aurait marqué un tournant dans la recherche d'immortalité : l'union avec le Dao matérialisée par l'immortalité devint dès lors un but scientifiquement accessible, une conséquence du procédé alchimique accompagné d'une persévérance spirituelle, et non un don inné. La particularité de la vision somatique de l'école Shangqing 上清派 (ch. v), un courant religieux fondé au IV siècle et marqué par l'individualisation des pratiques physiques, l'intériorisation de la méditation et la spiritualisation de la quête de l'immortalité, consisterait à identifier le corps individuel à un temple habité d'innombrables divinités, dont des personnes devenues immortelles. Or, au sein de l'école Lingba 霝寶派 (ch. vI) fondée au ve siècle, le prêtre devint la figure centrale de pratiques religieuses, son corps étant qualifié d' axis mundi entre le monde céleste des dieux et le monde terrestre des humains.

4 La période de la dynastie des Tang (618-907) fut non seulement l'âge d'or du taoïsme en général mais aussi celui du waidan 外丹, l'alchimie externe (ch. vII). Cet art de la confection d'élixirs spécifiques censés permettre d'inverser le processus cosmogonique et d'atteindre ainsi l'état originel atemporel, l'immortalité, aurait dû toutefois laisser place, en raison des décès causés par l'ingurgitation d'élixirs, à l'alchimie interne ou neidan 内丹 (ch. VIII), dès la dynastie des Song (960-1279). Désormais, pour atteindre ce même but, les partisans du neidan se focalisèrent sur le raffinage intérieur du corps matériel et spirituel. L'auteur semble alors reconnaître une de ces voies internes dans l'art dit de la chambre à coucher 房中術 (ch. Ix), dont l'essentiel consisterait à puiser le souffle, qi 氣, dans l'énergie sexuelle du partenaire de sexe opposé afin de prolonger au maximum la vie corporelle et de parvenir à l'immortalité. Une autre voie interne, ascétique, vit pourtant le jour au XII siècle. Ce fut grâce à l'école Quanzhen 全眞教 (ch. $\mathrm{x}$ ), le premier courant monacal taoïque, qui prôna le célibat, la meilleure façon de préserver l'essence vitale nécessaire pour atteindre le Dao. En outre, les arts martiaux taoïstes (ch. xI), tels que le taiji quan, créés indépendamment et devenus progressivement une composante du taoïsme, préconiseraient aussi le travail interne du souffle 内功. Quant à la médecine chinoise, zhongyi 中醫 (ch. XII), fondée éminemment sur le yinyang wuxing shuo, elle se préoccuperait de la circulation équilibrée des différents qi dans le corps pour une vie saine.

5 Ainsi, l'auteur présente l'évolution épistémologique et/ou herméneutique du corps humain, ainsi que des techniques conjointes de transformation corporelle et spirituelle, amorcées au sein de divers courants taoïstes. L'ouvrage permet alors au lecteur non 
seulement de parcourir plus de deux mille ans d'histoire du taoïsme, en prenant connaissance des questionnements actuels des milieux spécialistes et en percevant des liens causaux ou des influences réciproques entre divers courants taoïstes, mais aussi de découvrir des textes sources majeurs disciplinaires, accompagnés de leur version originelle. L'approche comparatiste, bien que parcellaire, des trois enseignements 三教, à savoir le confucianisme, le bouddhisme et le taoïsme, est également méritoire.

Néanmoins, les concepts somatiques du Liezi 列子, des wu 巫 et des fangshi 方士 auraient pu être intégrés dans la description de la période initiale du taoïsme afin d'éclaircir davantage l'évolution conceptuelle postérieure. En ce qui concerne les fondations du Tianshi dao, du Taiping dao et du Lingbao pai, une observation du contexte politique aurait élucidé leurs circonstances. Par ailleurs, l'historien des religions s'attendrait à une approche historico-critique des figures fondatrices des écoles taoïstes, au-delà de leurs hagiographies traditionnelles. Vis-à-vis de l'impact supposé du taoïsme sur la médecine chinoise (p.232), il faudrait rappeler que le yinyang wuxing shuo et la numérologie chinoise, dont l'origine respective n'est pas exposée dans l'ouvrage, formèrent un fonds commun pour le daojiao et le zhongyi. Du point de vue de l'histoire comparée des religions, la pratique de médiation du prêtre du Lingbao pai (p.133) et celle d'un chaman 巫 mériteraient une comparaison morphologique. La traduction du mot chinois «緣》 (lien, attachement, cause; skt. nidāna, pratyaya) par le terme bouddhique sanskrit «karma» (p.126) signifiant principalement «acte 業» s'avère inadéquate. Quant à la phrase citée (p. 204), le sinologue aurait pu proposer une traduction littérale plus conforme : «mieux vaut se sortir soi-même du cycle de la vie et de la mort 亦不如救自己» > 《mieux vaut se sauver ». Plus de précision sur l'école Jingming dao 淨明道 (p. 97), le rituel bouddhique (p. 130), le Guoyu 國語 (p. 230) et le (Huangdi neijing 黄帝内綵) Suwen 素問 (p. 232) aurait également facilité la lecture.

7 En outre, il reste quelques imprécisions éditoriales à rectifier. Avant tout, le sous-titre même de l'ouvrage doit être unifié entre "Chemins de découverte» (couverture et p. 285) et "Chemins de découvertes» (p. 5, 277). Deux patronymes et le titre d'un ouvrage sont à corriger : Mircea Iliade (p. 14) > Mircea Eliade ; Zhong Liquan (p. 116) > Zhongli Quan 鐘離權 (cf. p. 103, 160); Xuxian zhuan 續仙傳播 (p. 95) > 續仙傳. Certaines dates proposées sont inexactes: Zhou (1122-256) (p. 211) > Zhou (env. 1045-256 av. J.C.) ; Han (206 av. J.-C.-220 av. J.-C.) (p. 215) > Han (207 av. J.-C.-220 apr. J.-C.) (cf. p. 43) ; Liu Guizhen (1920-1943) (p. 225) > Liu Guizhen (1920-1983), etc.

8 Si le présent ouvrage, eu égard à l'absence de lecture historico-critique, ne peut être qualifié de recherche historique, il mérite cependant de figurer parmi les opera d'introduction disciplinaire thématique. Il offrira ainsi au lecteur une perspective chronologique et panoramique du taoïsme ainsi qu'une riche présentation de sources textuelles relatives à la perception somatique taoïque qui lui donneront assurément « le goût de les connaître et les lire davantage » (p. 15). 


\section{AUTEURS}

\section{KYONG-KON KIM}

Université de Strasbourg. 